\title{
Interoperable genome annotation with GBOL, an extendable infrastructure for functional data mining
}

\author{
Jesse C.J. van Dam ${ }^{1,{ }^{*}}$, Jasper J. Koehorst ${ }^{1,{ }^{*}}$, Jon Olav Vik ${ }^{2}$, Peter J. Schaap ${ }^{1}$, and Maria Suarez-Diez ${ }^{1}$ \\ ${ }^{1}$ Laboratory of Systems and Synthetic Biology, Wageningen University \& Research, Stippeneng 4, 6708 WE, Wageningen, The Netherlands \\ ${ }^{2}$ Centre for Integrative Genetics (CIGENE), Department of Animal and Aquacultural Sciences (IHA), Faculty of Life Sciences (BIOVIT), Norwegian University of Life Sciences \\ (NMBU), PO Box 5003, Ås, Norway \\ *Authors contributed equally
}

\section{Background}

A standard structured format is used by the public sequence databases to present genome annotations. A prerequisite for a direct functional comparison is consistent annotation of the genetic elements with evidence statements. However, the current format provides limited support for data mining, hampering comparative analyses at large scale.

Results

The provenance of a genome annotation describes the contextual details and derivation history of the process that resulted in the annotation. To enable interoperability of genome annotations, we have developed the Genome Biology Ontology Language (GBOL) and associated infrastructure (GBOL stack). GBOL is provenance aware and thus provides a consistent representation of functional genome annotations linked to the provenance.

GBOL is modular in design, extendible and linked to existing ontologies. The GBOL stack of supporting tools enforces consistency within and between the GBOL definitions in the ontology (OWL) and the Shape Expressions (ShEx) language describing the graph structure. Modules have been developed to serialize the linked data (RDF) and to generate a plain text format files. Conclusion

The main rationale for applying formalized information models is to improve the exchange of information. GBOL uses and extends current ontologies to provide a formal representation of genomic entities, along with their properties and relations. The deliberate integration of data provenance in the ontology enables review of automatically obtained genome annotations at a large scale. The GBOL stack facilitates consistent usage of the ontology.

RDF | Genome | Annotation

Correspondence: maria.suarezdiez@wur.nI

\section{Background}

Advances in sequencing technologies have turned genomics into a data-rich scientific discipline in which the total assembled and subsequently annotated sequence data doubles every 30 months (1). To support the growth in data throughput, automated annotation algorithms have become an indispensable supplement to manual annotation $(2,3)$ and currently, automatic annotations in the UniProt database outnumber manual annotations 100-fold (4).
Functional genome comparison has been used to identify diagnostic markers, to develop effective treatments, and to understand genotype-phenotype associations (5-7). The volume and heterogeneity of genome annotation data has created a unique type of big data challenge, namely how to transform computational predicted annotations into actionable knowledge. Tapping into these available resources is only efficiently done by computational means and requires a consistent interlinking of data so that data becomes Findable, Accessible, Interoperable and Reusable (FAIR) (8).

The format for sharing of public genome sequence annotation data has been developed and is maintained by the International Nucleotide Sequence Database Collaboration (INSDC) a long-standing foundational initiative that operates between the DDBJ, EMBL-EBI and NCBI public repositories. However, tradeoffs between simplicity, human readability and representational power left little support for interoperability, i.e. the ability of computer systems to directly make use of information. The /inference qualifier (9) provides a structured description of evidence that supports feature identification or assignment. Thus, within the standard formats, data provenance of computational annotations could be stored under this optional inference tag but this tag is not designed to be used for contextual, element-wise provenance.

Currently, most annotations rely on computational predictions of structure or function, and the choice of thresholds for confidence scores becomes a key consideration. Tracking the provenance of genome annotations becomes essential for scientific reproducibility and to enable critical reexamination of analyses. However, such meta-analysis is currently very time-consuming. Efficient meta-analysis would require a framework able to accommodate the various types of annotations (e.g. gene prediction, homology, protein domains) directly linked to the supporting statistical evidence. Presently, no machine-readable infrastructure exists to directly query genome annotations linked to the historical and contextual provenance. The World Wide Web consortium provides the Semantic Web and the Resource Description Framework (RDF) data model, supporting these requirements. For RDF, ontologies are essential as they provide consistency in the meaning of data elements and in the relationship between them (10). 
In this respect, ontologies already exist for various aspects of biology (11). The Sequence Ontology (SO) (12) was presented over 12 years ago and was designed as a complete terminology of unambiguous terms related to genetics. However, it was never intended to function as a file format or database schema, and provides no support for linked sets of data attributes. Furthermore, it has limited support for storing based-on provenance except for some experimental codes. FALDO's (13) only purpose is to unambiguously store genetic locations on a sequence. The Synthetic Biology Open Language (SBOL) (14) was successfully designed to describe complete synthetic constructs and the interactions between each of the elements. None of these standards were designed to consistently store feature predictions with evidence provenance and therefore none of these tools provides a complete representation of the genomic information linked to the provenance it is based on.

To meet the requirements and to ensure interoperability of computational predictions, we developed an extendable provenance-centered infrastructure for interoperable genome annotations. The here presented infrastructure consists of two main elements; Firstly, the Genome Biology Ontology Language (GBOL), which directly integrates evidence provenance for the whole dataset and for each included element (dataset- and element- wise provenance). Secondly, the "GBOL stack" of enforcing tools facilitates the consistent usage of ontologies. GBOL is modular in design, extendible and linked to existing ontologies. Empusa has been developed as part of the GBOL stack to ensure consistency within and between ontology (OWL), the API and the Shape Expressions (ShEx) describing the graph structure. This enables the use of SPARQL queries to include contextual details in large scale functional analyses. Modules have been developed to serialize the linked data (RDF) and to generate a plain text format files.

\section{Results}

Ontology structure. GBOL is a genome annotation ontology developed for the application of semantic web technologies in genome annotation and mining. As such GBOL provides the means to consistently describe computationally inferred genome annotations of biological objects typically found in a genome sequence annotation data file in the public repositories. Additionally, it can describe the linked data provenance of the extraction process of genetic information from genome sequences.

An overview of the structure of GBOL is shown in Figure 1. The ontology contains 251 classes that can be categorized into 6 broad domains (Table 1). In GBOL, sequences have features, which in turn have genomic locations on the sequence. The authority of this relationship is derived from the data provenance that captures both the statistical basis of each individual annotation (element-wise provenance) as well as the programs and parameters used for the complete set of sequences under study (dataset-wise provenance). All annotations for a given sequence can be packed into a single entity called a document.
Table 1. Overview of domains, classes and properties described by the the GBOL ontology. Note that some properties might be in multiple sub domains.

$\begin{array}{llll}\text { Sub domain } & \text { Classes } & \begin{array}{l}\text { Properties } \\ \text { Genomic locations }\end{array} & \begin{array}{l}\text { Value } \\ \text { sets }\end{array} \\ \begin{array}{l}\text { Genes } \\ \text { transcripts and features }\end{array} & 114 & 17 & 1 \\ \text { Document structure } & 27 & 133 & 17 \\ \text { Dataset-wise provenance } & 22 & 54 & 7 \\ \text { Element-wise provenance } & 5 & 9 & 0 \\ \text { BIBO } & 59 & 90 & 2\end{array}$

Design principles. GBOL was developed focussing on its function as as file format and as database schema and has the following design principles: modularity, human readability, and annotation. These principles ensure that the ontology can be easily extended (16).

Modularity: The number of classes in the main class tree is kept as small as possible and elements within the data are described with attributes when possible. Furthermore, classes are included in the main class tree only when there are unique properties in a class or in one of the sibling classes. This approach ensures that sub-ontologies can be managed as separate entities within the main ontology and that we can use existing ontologies. As an example the class RegulationSite has an attribute regulatoryClass, which denotes the type of regulation with a separate set of classes of which all are instances of the regulatory class.

To further simplify the ontology, every attribute is defined as a direct property within the class that links to either a string, an integer, another object or a class in an enumeration set. For each class in which the attribute is used, an 'all values from' axiom is used, with an optional minimal and/or maximal cardinality constraint. The 'all values from' axiom enforces all referenced objects to be of the expected type, which is not the case with the 'some values of' axiom and therefore we excluded the use of the 'some values of' axiom. This approach is fundamentally different from the principle used in the SO, in which attributes are defined using the 'has quality' property in combination with the 'some values of' axiom that references to a class.

Human Readability: All names within the ontology adhere to a set of basic principles to increase (human) readability of the ontology. All class names represent the underlying biological concept as closely as possible avoiding the use of unreadable numbers. All classes start with uppercase whereas properties start with lowercase. All words are spelled out, and white spaces are left out of the names, instead the next word starts with uppercase. In this way, the class 'exact position' becomes 'ExactPosition' and the property 'regulatory class' becomes 'regulatoryClass'. Furthermore, where possible, the names are shortened with abbreviations, as long as they remain understandable for a human reader (e.g. XRef instead of CrossReference).

Annotation: All classes and terms within the ontology are 


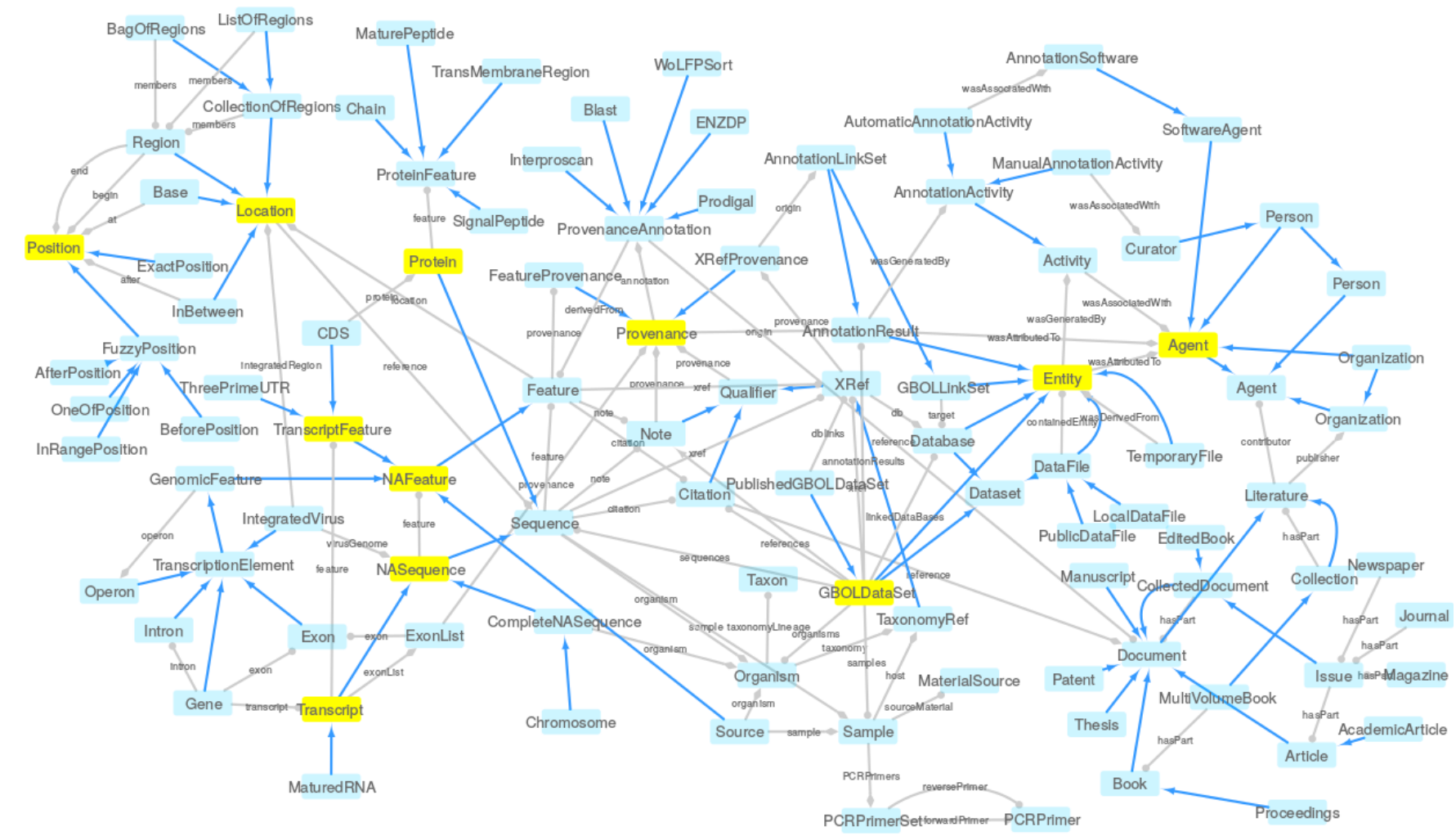

Fig. 1. The GBOL ontology structure: Network based view generated using RDF2Graph(15) the GBOL core ontology. Nodes represent types. Blue edges represent subClassOf relationships whereas grey edges represent unique type links. A unique type link is defined as a unique tuple: type of subject, predicate, (data)type of object. Arrow heads indicate the forward multiplicity of the unique type links: 0.1 and $1 . .1$ multiplicities are indicated by diamonds; $0 . . \mathrm{N}$ and $1 . . \mathrm{N}$ multiplicities are indicated by circles. Neighbourhood of nodes marked in yellow is further expanded in Figures 4-8

annotated with a short definition; an optional comment with additional usage information; an optional editorial comment relating to the development of the ontology itself; an optional $d d b j$ label indicating the presence in the GenBank standard; and an optional SKOS (17) exact match to relate classes to terms in existing ontologies.

The GBOL infrastructure. An infrastructure enabling interoperable genome annotations integrated with provenance requires the following characteristics: i) An OWL (18) encoded definition of an ontology. ii) An infrastructure to enhance and simplify its usage, consisting of an interface (API) that allows to use Java and R. iii) A file format that can be obtained from serializing the linked data (RDF) using a lightweight Linked Data format (JSON-LD) (19) which is subsequently serialized as YAML (20). This format mimics the layout of the current format for sharing of public genome sequence annotation data, but has integrated support to add additional information. iv) A ShEx definition for data conformance validation to enhance data consistency (21). And v) a tool to convert existing GenBank and EMBL format files into the GBOL format.

GBOL data can be stored in any of the linked data formats (RDF), such as Turtle. The generated API can be used to access the genomic information encoded within the GBOL format, which includes a data consistency validation. The API directly reads from and directly modifies the RDF data structure upon usage of any of the data model functions. This enables the usage of SPARQL within the client code, which can

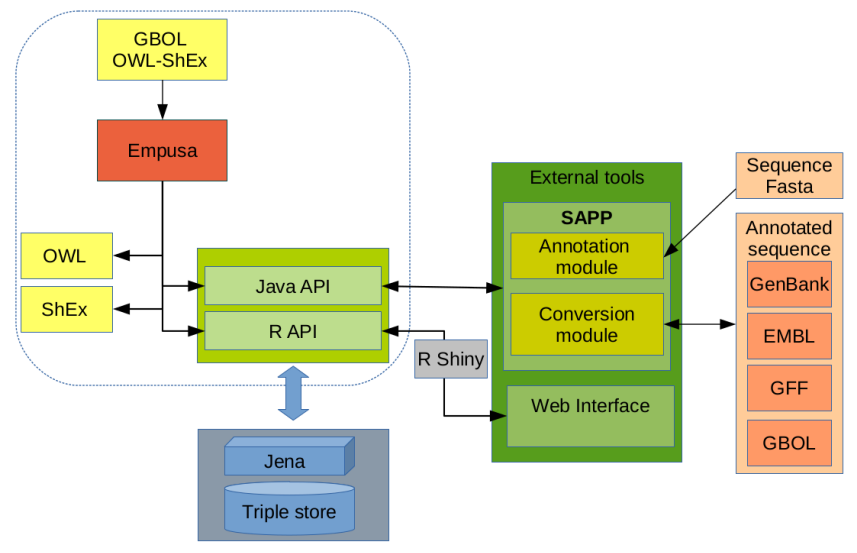

Fig. 2. Schematic of an interoperable provenance centered genome annotation pipeline. The GBOL stack (dashed box) provides the Genome Biology Ontology Language (GBOL) (Yellow) and associated infrastructure to keep it consistent and extendable (Empusa). The SAPP module functions as an interface for (standardly used) genome annotation tools. Using the JAVA API, SAPP retrieves raw genome data from the triple store, runs genome annotation tools in batch and uses the GBOL ontology to automatically store their predictions and associated data provenance directly as RDF triples in the triple store database (Blue). Stored predicted functional annotations, data provenance and linked meta-data can be queried within JAVA and R with SPARQL and by using a web interface (Green). Parsers have been developed for conversion of annotation files in standardly used formats (Orange).

run a SPARQL query and directly use the resulting objects nodes in the API. Moreover, the RDF data can be structured into a tree with the JSON-LD framing API into JSON-LD, which, in turn, can be further serialized as YAML resulting in a human readable format for sharing of public genome se- 
quence annotation data. By addition of standard annotation tools, the GBOL stack can be at the core of a provenancecentered genome annotation framework (Figure 2).

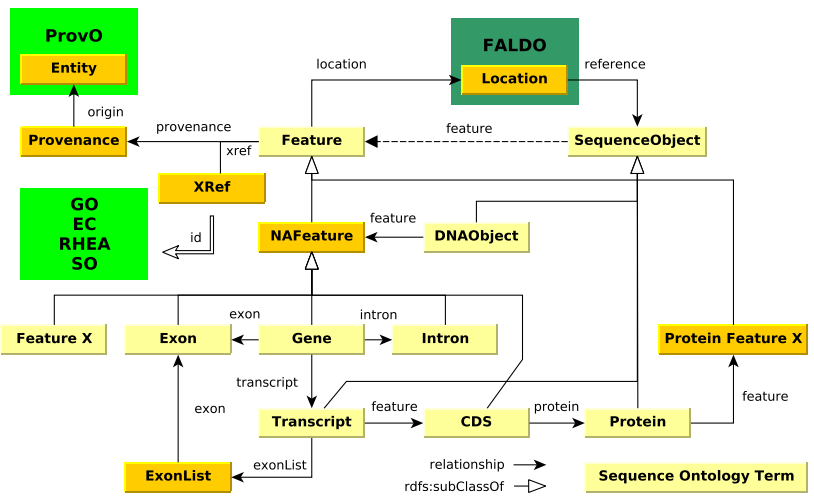

Fig. 3. Embedding of the GBOL ontology with already existing ontologies. FALDO, Provo, GO, EC, RHEA and SO are existing ontologies. Classes are in yellow and an explanation is provided in the main text.

Embedding with other ontologies. GBOL is embedded in the corpus of currently developed web technologies and when possible we have integrated existing ontologies such as: FALDO (13), PROV-O (22), SO (12), SBOL (14), BIBO (23), WikiData (24), FOAF (25), Gene ontology (GO) (26) and the Evidence ontology (27) as depicted in Figure 3. Annotation of genomic location is inspired by FALDO ontology, although several elements had to be modified. The PROV-O ontology was used and extended to store data provenance. Whenever applicable, we added a cross-link to exact matching terms within the FALDO, SO and SBOL ontologies. Identification of persons and institutions is done through the FOAF ontology and BIBO is used to identify publications. GBOL does not represent a vocabulary to describe genetic, molecular or cellular functions. Instead, terms can be crossreferenced to the many vocabularies that provide functional descriptions to the (products of) genetic elements, such as Gene Ontology, Enzyme commission (EC) numbers, and the CHEBI and RHEA databases $(28,29)$, among others.

Key GBOL classes. Common elements in genome annotations include different classes of DNA molecules such as chromosomes, plasmids and contigs, genes, transcripts, exons, introns, proteins, protein domains and functional annotations. The following sections summarize the key classes of the ontology. An extensive description for each element can be found in the documentation available at http://gbol . life/0.1/.

Genomic locations: Genomic locations of all features in GBOL is captured with the Location, Position and StrandPosition classes, which are inspired by the FALDO ontology and represented in Figure 4. The Location and its subclasses together with the StrandPosition define an interval on the Sequence, whereas Position defines a single position in a sequence. A location can be either: i) A region which has begin and end positions; ii) A collection of regions (ordered or unordered); iii) A single base at a given position; or iv) an In-
Between location denoting a location between two bases after the base of which the position is given. Each region, base and in-between location can be defined to be located on the forward, reverse or both strands, although no strand should be specified if the sequence is a single stranded DNA sequence or a protein sequence. It should be noted that elements of a collection of regions can be located on different sequences. This can be used to encode cases in which an otherwise indistinguishable genetic element is located on multiple chromosomes.

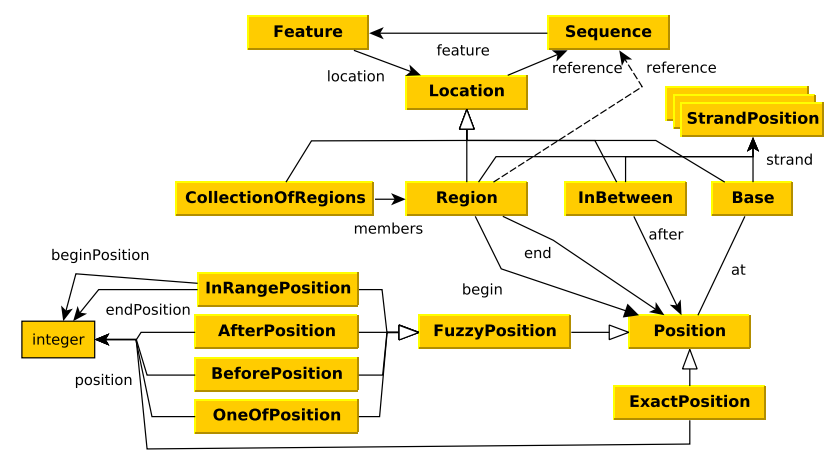

Fig. 4. Graphical view of the GBOL ontology for genomic locations. An explanation of the classes is provided in the main text.

Exactly known positions can be indicated using the ExactPosition class containing the position property. Otherwise a not exactly known position, also called fuzzy position, can be indicated using either the BeforePosition class containing the position property, the AfterPosition class containing the position property, the InRangePosition class containing the beginPosition and endPosition properties or the OneOfPosition class containing multiple position properties.

Genes, transcripts and other commonly encountered genomic features: GBOL has a consistent model for storing genes, exons, (alternatively spliced) transcripts, coding sequences and proteins. Central to this model is the Sequence class that can have multiple annotations represented in the Feature class. An overview is provided in Figure 5.

In GBOL a sequence can be specified as a nucleic acid (NA) or a protein sequence. The sequence is attached to the $\mathrm{Se}$ quence class via the sequence property, provided in the DNA, RNA or protein encoding standard. NA-sequences can represent transcripts or other elements such as chromosomes, plasmids, scaffolds, contigs or reads. No distinction is made between DNA and RNA and the strandType denotes that it is either a double or single stranded DNA or RNA. As indicated in Figure 5 the type of sequence determines the features it might be associated to (ProteinFeature, NAFeature or TranscriptFeature),

Typically, each GBOLDocument contains one or more NASequences (e.g. Chromosome, Contig, $m R N A$ ), which can have multiple features including all gene, exon, intron, sequence variations, and structural, regulatory and repeat annotations. Each gene is linked to its associated exons, introns and transcripts. Due to alternative splicing a gene can have multiple transcripts. Each transcript has its own unique list of exons, which is linked through the exonList and associated exonList 


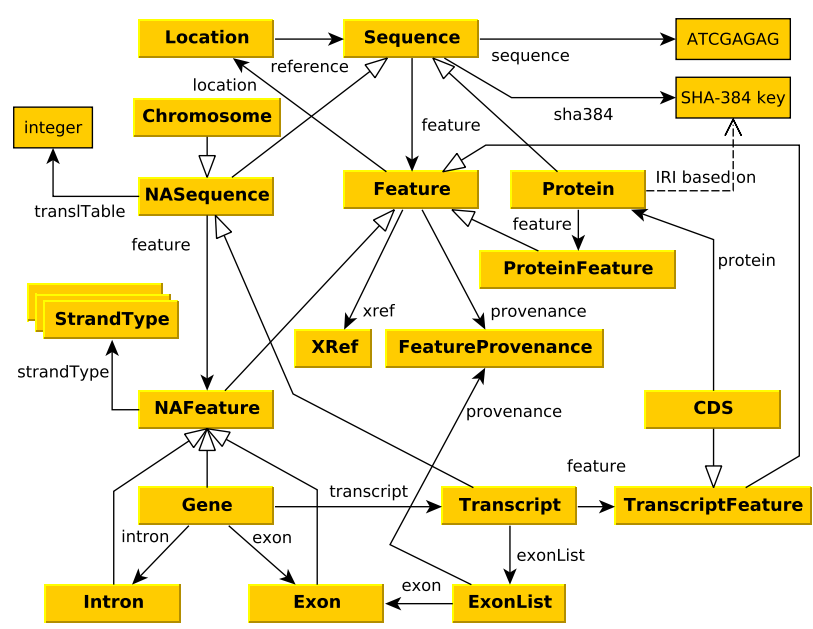

Fig. 5. Graphical view of the GBOL ontology for genes, transcripts and other commonly encountered genomic features. An explanation of the classes is provided in the main text

class to all associated exons. A transcript can be either a mRNA, ncRNA, rRNA, tmRNA, tRNA, precursor RNA or a miscellaneous RNA. The type of transcript determines the associated features: mRNA transcripts can have features linked to coding sequence (CDS), 5' -UTR, 3'- UTR and poly A tail. The mRNA translation table is defined with the translTable property from the parent sequence. The association between CDS and the encoded protein is preserved and information about the translation is stored if it is different from the default translation (for example, use of alternative stop codons).

Each protein has a unique IRI (http://gbol.life/0.1/protein/<SHA-384>) based on the SHA-384 hash of its sequence. This makes it possible to combine protein information from heterogeneous sources, as a protein can be associated to several CDS features. All information related to the protein which is unique to the genome (such as location) should be stored in the CDS feature. Protein annotation features may include, among other, conserved regions, protein domains, binding sites, 3D structure, signal peptides, transmembrane regions, and immunoglobulin regions. Operons can be defined with the Operon feature, to which other genomic features, such as genes, can be associated. Additionally, viral genome integration can be denoted using the IntegratedVirus feature.

Provenance related classes. Three types of provenance can be distinguished. Metadata refers to the owners of the samples, the biological origin, culture conditions etc. Datasetand element- wise provenance pertain to the annotation process. All data within a single data collection stored in GBOL is based on the GBOLDataSet, which holds among other, references to all included samples, sequences, organisms, annotation results and linked databases. An overview of the document structure is given in Figure 6.

A sequence originates from a sample and samples are related to one or multiple organisms. The sample property which links to the Sample class describes where, when, how, by whom and from what the sample was collected. The

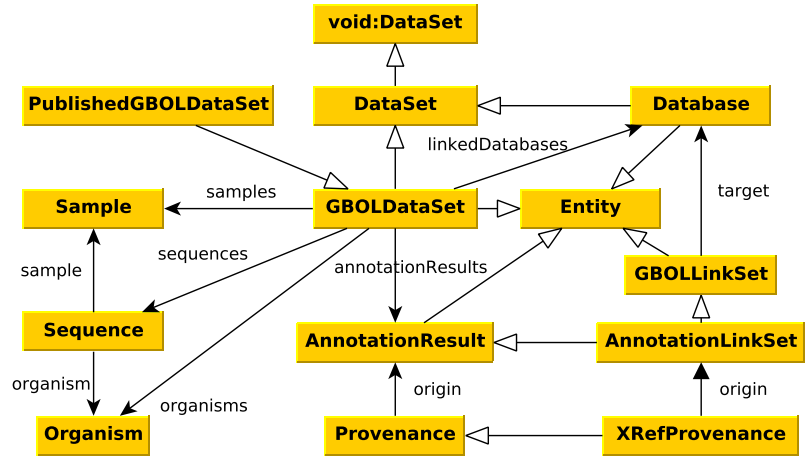

Fig. 6. Graphical view of the GBOL Document structure. An explanation of the classes is provided in the main text

fields follow the GenBank format. The organism property describes the taxonomic reference, its scientific name and its taxonomic lineage.

All annotations made within the GBOLDataSet have associated provenance and should originate from one of the listed annotation results, so that correspondence with originating databases is preserved. The Database and the GBOLDataSet classes are both sub classed from the void ontology, Dataset class contains a general description, including among other title, description, comment, license, version, data download address, SPARQL endpoint URI, and URL encoding.

Dataset-wise provenance: Storage of the dataset-wise provenance is based on the PROV-O ontology in which the Entity, Agent and Activity classes are central. An activity can use and generate entities, which are executed (wasAssociatedWith) by an agent. As a result, an entity can be attributed to an agent. The GBOLDataset, AnnotationResult, GBOLLinkSet and Database classes (indicated in Figure 6 and 7) are subclasses from the PROV-O ontology Entity class, so that for each of these objects provenance on how, when and by whom they were created can be associated.

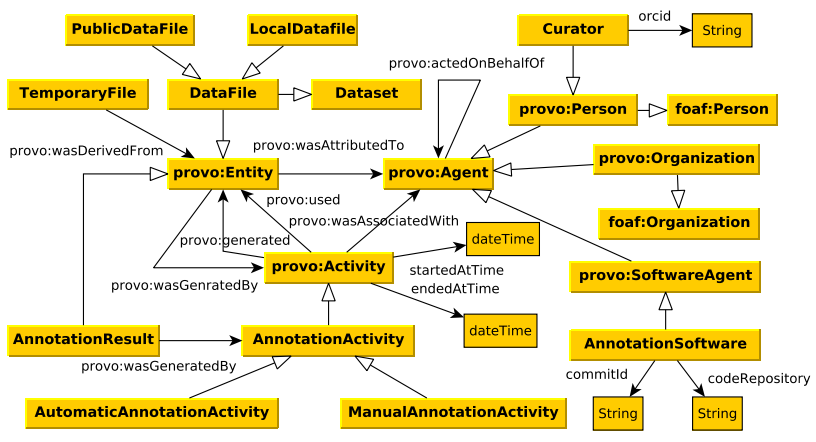

Fig. 7. Graphical view of the GBOL Dataset-wise provenance. An explanation of the classes is provided in the main text

In GBOL an Entity is either a file or an annotation result. The annotation result is a set of triples contained within a GBOL document, whereas a file represents a physical file either on a computer or network. An agent can either be a curator, person, organization or annotation software. For the annotation software a version and code repository with associated commit identifier is included to enable univocal identifica- 
tion. For a curator, an ORCID (30) must be specified so that each curator can be uniquely identified together with his/her organization. Both Person and Organization are sub-classed from the FOAF ontology to include additional information such as name and email address.

Within GBOL, each activity is an annotation activity, which can be either an automatic process or a manual curation activity, with a start and end time. An automatic annotation must be associated with a software agent and the set of parameters used must be specified including the corresponding input and/or output files. Finally, manual curation must be associated with a curator.

Element-wise provenance and qualifiers: In addition to the dataset-wise provenance, GBOL is able to capture an additional layer of element-wise provenance, as the provenance of all the annotation in GBOL is captured per property per feature with the FeatureProvenance, as shown in Figure 8. For properties that could have items from multiple sources, we have defined the Qualifiers, each with its own associated provenance. A qualifier can either be a citation, note or cross reference (indicated by $x r e f$ ). A citation can hold a reference to literature encoded with the BIBO ontology.

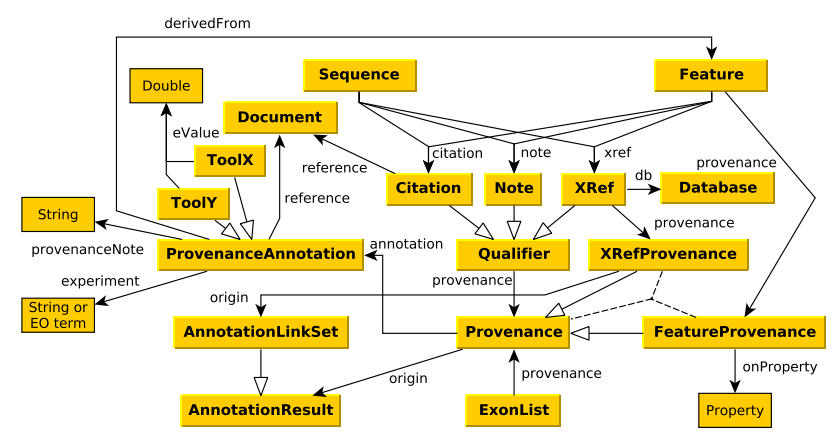

Fig. 8. Graphical view of the GBOL element-wise provenance. An explanation of the classes is provided in the main text

Annotations are linked to the provenance object either through the provenance property of the qualifiers or the onProperty property of the Provenance feature. The provenance object links to both the dataset-wise provenance and the element-wise provenance. The origin links the provenance with the dataset-wise provenance (AnnotationResult), which includes among other the creation time, identity of the creating agent and the used parameters, as previously mentioned. The annotation links to the element-wise provenance (ProvenanceAnnotation), which includes: A free text note to describe the annotation; A list of references supporting the note; An experimental code, preferably from the Evidence Ontology to qualify the evidence supporting the conclusion; An optional derivedFrom that links to other features on which it is based.

Finally, each annotation tool generates its own evidence statements, often embedded in a statistical framework, characteristic of the algorithmic approach taken, such as p-values, bit scores, matching regions or any other scoring system. To store tool specific confidence scores, a subclass of the ProvenanceAnnotation class can be created. Some example classes include Blast, HMM and SignalP associated with the output of corresponding tools (31-33) However, these classes are not part of the GBOL ontology itself.

Empusa. During the development of the standard, difficulties were encountered in managing the large set of properties and structures in the OWL and ShEx definitions and the API needed to encode the annotation information in conjunction with the associated provenance. Moreover, Analyses of various public repositories have shown that inconsistent, nonenforced usage of ontologies leads to mismatches between the descriptive OWL file and the actual content (15). In order to shorten the development cycle and to maintain consistency within and between the OWL and ShEx definitions and the API, a standalone tool was developed named Empusa. The input definition of Empusa is a combination between OWL and a simplified version of ShEx, which can be edited within Protégé (34). The classes are defined in OWL, whereas the properties are defined in each class under the annotation property 'propertyDefinitions' encoded within a simplified format of the ShEx standard. Additionally predefined value sets (for example all regulatory types) can be defined by adding a subclass to the EnumeratedValueClass. Each subclass of the value set is represented as one element within the value set. As standalone tool, Empusa can automatically and consistently generate an OWL and a ShEx definition, ontology documentation in markdown, an API, a JSON-LD framing file and a visualization. Empusa uses parts of the RDF2Graph tool (15) to generate a representation that can be subsequently used to generate a visualization within Cytoscape (35). This allows users to browse the complete ontology intuitively.

\section{Discussion}

Comparative genome analysis is essential to understand the mechanisms underlying evolution and adaptation. Ideally, comparative genomics should be performed at the functional level, as this is highly scalable and more resistant to phylogenetic distances (36). However, as functional annotation is performed in a non consistent manner the current practical level of interoperability is at the sequence level. Many tools exists to obtain orthologous clusters which are shaped by a generalised acceptance threshold for similarity and alignment length which is a trade-off between sensitivity and false discovery $(37,38)$. At large scales these analysis are hampered by the high computational cost for finding bi-directional best matches. We have shown (36) that functional comparison, based on consistently annotated protein domains, provides a fast, efficient and scalable alternative .

The prerequisite of a direct comparative functional analysis is consistent annotation of the genetic elements with evidence statements. Recording the provenance allows class-specific cut-offs for each individual annotation. Element-wise provenance enhances the re-usability of the annotations, and allows the development of methods to combine evidence statements, often derived from complex statistical frameworks, into confidence statements. Element-wise provenance also enables a 
quick re-evaluation of evidence, for instance by using a tunable cut-off score.

GBOL has been developed to explore available genome sequences using the mining possibilities of linked data. As a result, GBOL has evolved to consistently capture annotation data generated by the Semantic Annotation Platform with Provenance (SAPP), available at http://semantics. systemsbiology.nl. Previous versions of the GBOL ontology have been used to compare 432 Pseudomonas strains through integration of genomic, functional, metabolic and expression data (39). Here GBOL was essential to capture, store and interlink the genomic and functional annotation data. Strikingly, over 432 Pseudomonas strains, consistent de-novo annotation yielded 838 additional GO-terms and 146 additional protein domains which would not have been identified using the original gene predictions. In addition to determining the functional pan- and core genome of a species, comparative genomics also enables the investigation of genotype-phenotype associations. In (40) we consistently functionally annotated and compared 80 publicly available mycoplasma genomes. The resulting semantic framework allowed us to efficiently query for functional differentiation of various mycoplasma species in relation to host specificity and phylogenetic distance.

Consistent functional annotation within a semantic framework requires a standardised ontology for the annotated elements and the associated based-on provenance. Linked data ensures that queries can be performed, mining multiple sequences at once, thereby providing a scalable alternative for large scale genome comparisons. The GBOL stack provides the ontology and corresponding API that enables the incorporation of functional annotation and provenance reducing complexity and is the outcome of efforts in a number of studies related to functional comparative genomics. Currently the GBOL stack is being used in various collaborative projects to handle genomic data of organisms across all domains of life (41-44).

GBOL has been primarily designed to handle genomic annotation. However, it has been designed in a modular and extensible manner so that in the future it can be extended to host other omics data types as proteomics and transcriptomics. The modular design of GBOL ensures that other ontologies can be incorporated and managed as separate entities. For instance, the majority of the feature and sequence classes within GBOL can be connected with those from the Sequence Ontology and are therefore linked with the skos:exactMatch predicate. The major difference between GBOL and SO is that $\mathrm{SO}$ has been defined as vocabulary of terms related to genetic elements, whereas the GBOL classes have been designed to describe genetic annotation and elements located on a sequence and is inspired on the principles of the GenBank format. However, still a number of features in the SO are not currently available in GBOL and future work should focus on including them. Another possible extension would be to link to other Minimum Information Standards like MIGS and extensions thereof (MIMARKS, MIxS) $(45,46)$ and cross domain experiment reporting standards like ISA-tab (47). Other possible extensions relate to the development of the subontologies GBOL links to. For instance, BIBO is used to store information on literature references, however the OWL ontology file of BIBO has to be further improved, as it does not specify to which classes all of the properties should belong. Therefore we have chosen to include a less consistent representation of the properties by adding all properties to the root class bibo:Document.

Empusa, a core part of the GBOL stack, ensures the correct usage of the ontology through the provided R and JAVA API. We have ensured that Empusa can be used independently of GBOL (documentation available at http://gbol. life) and therefore can be used to develop new ontologies combined with an automatically generated API and documentation. This reduces the complexity and time to extend and develop ontologies with corresponding API's and ensures consistent and correct usage of a defined ontology.

\section{Conclusions}

Large scale analysis of heterogeneous biological data is hampered by lack of interoperability. To improve the exchange of information formalized information models are required. GBOL provides a formal representation of genomic entities, their properties and relations. The GBOL Stack provides a framework to enforce consistent and correct usage of GBOL. The semantic basis and the integration of provenance enables FAIR genome annotations, thereby unlocking the potential of functional genome annotation data.

\section{Methods}

The GBOL ontology is OWL encoded and a ShEx schema is provided. All supporting software (Java and R API, Empusa) are written in Java with Gradle as build system. We use JENA (48) for handling and loading the RDF data into a triple store. Protégé was used for editing the ontology(34).

Storage of the genomic location is inspired by FALDO, although several elements had to be modified e.g. to account for features that start and end on different sequences. Differences include: i) StrandPosition is not subclassed from Position. Instead, an additional property is added to the region, base and InBetween location, this is done because these location object types can have both a strand position and an index position on the sequence. ii) The reference property is not part of a Position, but of a Location, because a location that starts on one sequence and ends on another sequence is an undefined sequence. iii) The BaseLocation and the InBetweenLocation classes have been added to the ontology. iv) The BaseLocation, InBetweenLocation, CollectionOfRegions and Region are children of the Location class, such that the rest of the ontology can incorporate these classes. v) The before and after positions have been explicitly defined to include their semantics. vi) The classes sub-classed from FuzzyPosition have an integer to denote the position and do not point to another position object, which could allow for arbitrary complex location denotations. vii) The $\mathrm{N}$ - and C-terminal positions have been removed and all indexes are counted from 
bioRxiv preprint doi: https://doi.org/10.1101/184747; this version posted September 5, 2017. The copyright holder for this preprint (which was not certified by peer review) is the author/funder, who has granted bioRxiv a license to display the preprint in perpetuity. It is made available under aCC-BY-NC-ND 4.0 International license.

the N-terminal side. Counting from the C-terminal side can be calculated based on the sequence length. vii) The reflective properties beginOf and endOf have been removed, because a position can also be referenced by the added base location. For consistency we have redefined all FALDO elements within our own namespace.

Cross-links to exact matching terms from other ontologies (such as FALDO, SO and SBOL ) where added using skos:exactMatch. Additionally, several properties within the ontology point to existing ontologies, for instance: i) The signalTarget property of SignalPeptide, the modificationFunction of ModifiedResidue and the organelle of Sample are interlinked with GO terms. ii) The experiment property of ProvenanceAnnotation, which denotes upon which evidence the annotation is based on, should point, where possible, to a term within the Evidence Ontology . iii) The residue property of ModifiedResidue must point to a term within the Protein Modification Ontology (49). iv) GBOL includes the GO terms for tissueType of the Sample class and points, when possible, to a term within the BRENDA Tissue and Enzyme Source Ontology (50).

The source file of the ontology encoded in the Empusa and associated generated OWL definition, ShEx schema and visualization for Cytoscape available at http://www . git lab.com/GBOL under the MIT license. The generated Java and R API are available at https://gitlab.com/ gbol/GBOLapi and https://gitlab.com/gbol/ RGBOLApi under the MIT license. The conversion module, which is part of SAPP, is available at http:// www.gitlab.com/SAPP/conversion under the MIT license. The supporting Empusa code generator is available at http://www.gitlab.com/Empusa under the MIT license. All projects are coded in Java and are based on the Gradle build system. All terms are resolvable and can be browsed for at the associated website http://gbol. life/0.1/.

\section{ACKNOWLEDGEMENTS}

We thank Benoit Carreres for helpful design discussions. This work has received funding from the Research Council of Norway, No. 248792 (DigiSal) and from the European Union FP7 and H2020 under grant agreements No. 305340 (INFECT), No. 635536 (EmPowerPutida) and No. 634940 (MycoSynVac).

\section{Bibliography}

1. ENA. ENA european nucleotide archive statistics. http://www.ebi.ac.uk/ena/ about/statistics, 2017.

2. Tatiana Tatusova, Michael DiCuccio, Azat Badretdin, Vyacheslav Chetvernin, Eric P Nawrocki, Leonid Zaslavsky, Alexandre Lomsadze, Kim D Pruitt, Mark Borodovsky, and James Ostell. Ncbi prokaryotic genome annotation pipeline. Nucleic acids research, 44 (14):6614-6624, 2016.

3. Torsten Seemann. Prokka: rapid prokaryotic genome annotation. Bioinformatics, 30(14): 2068-2069, 2014.

4. The UniProt Consortium. UniProt: a hub for protein information. Nucleic acids research, 43 (Database issue):D204-12, 2015. ISSN 1362-4962. doi: 10.1093/nar/gku989.

5. Bas E. Dutilh, Lennart Backus, Robert A. Edwards, Michiel Wels, Jumamurat R. Bayjanov, and Sacha A. F. T. van Hijum. Explaining microbial phenotypes on a genomic scale: GWAS for microbes. Briefings in Functional Genomics, 12(4):366-380, July 2013. ISSN 20412649, 2041-2657. doi: 10.1093/bfgp/elt008.

6. David N. Cooper, Michael Krawczak, Constantin Polychronakos, Chris Tyler-Smith, and Hildegard Kehrer-Sawatzki. Where genotype is not predictive of phenotype: towards an understanding of the molecular basis of reduced penetrance in human inherited disease. Human Genetics, 132(10):1077-1130, October 2013. ISSN 1432-1203. doi: 10.1007/s00439-013-1331-2.

7. Jessica Alföldi and Kerstin Lindblad-Toh. Comparative genomics as a tool to understand evolution and disease. Genome Research, 23(7):1063-1068, July 2013. ISSN 1088-9051. doi: $10.1101 /$ gr.157503.113
8. Mark D Wilkinson, Michel Dumontier, IJsbrand Jan Aalbersberg, Gabrielle Appleton, Myles Axton, Arie Baak, Niklas Blomberg, Jan-Willem Boiten, Luiz Bonino da Silva Santos, Philip E Bourne, and Others. The FAIR Guiding Principles for scientific data management and stewardship. Scientific data, 3:160018, 2016.

9. /inference qualifier. http://www. insdc.org.

10. Robert Hoehndorf, Paul N. Schofield, and Georgios V. Gkoutos. The role of ontologies in biological and biomedical research: a functional perspective. Briefings in Bioinformatics, 16 (6):1069-1080, November 2015. ISSN 1467-5463. doi: 10.1093/bib/bbv011.

11. Jonathan B. L. Bard and Seung Y. Rhee. Ontologies in biology: design, applications and future challenges. Nature Reviews Genetics, 5(3):213-222, March 2004. ISSN 1471-0056. doi: $10.1038 / \mathrm{nrg} 1295$.

12. Karen Eilbeck, Suzanna E Lewis, Christopher J Mungall, Mark Yandell, Lincoln Stein, Richard Durbin, and Michael Ashburner. The Sequence Ontology: a tool for the unification of genome annotations. Genome biology, 6(5):R44, 2005. ISSN 1465-6914. doi: 10.1186/gb-2005-6-5-r44.

13. J. Bolleman, C. J. Mungall, F. Strozzi, J. Barran, M. Dumontier, R. J. P. Bonnal, R. Buels, R. Hoendorf, T. Fujisawa, T. Katayama, and P. J. a. Cock. FALDO: A semantic standard for describing the location of nucleotide and protein feature annotation. Journal of Biomedical Semantics, pages 1-19, 2016. ISSN 2041-1480. doi: DOI10.1186/s13326-016-0067-z.

14. Michal Galdzicki, Kevin P Clancy, Ernst Oberortner, Matthew Pocock, Jacqueline Y Quinn, Cesar A Rodriguez, Nicholas Roehner, M, Mandy L Wilson, Y Wilson, Laura Adam, J Christopher Anderson, Bryan A Bartley, Jacob Beal, Deepak Chandran, Deepak Ch, Joanna Chen, Ran, Douglas Densmore, Drew Endy, Raik Grünberg, Jennifer Hallinan, Nathan J Hillson, Jeffrey D Johnson, Allan Kuchinsky, Matthew Lux, Goksel Misirli, Jean Peccoud, Hector A Plahar, Evren Sirin, Guy-Bart Stan, Alan Villalobos, Anil Wipat, John H Gennari, Chris J Myers, and Herbert M Sauro. The Synthetic Biology Open Language (SBOL) provides a community standard for communicating designs in synthetic biology. Nature Biotechnology, 32(6):545-550, 2014. ISSN 1087-0156. doi: 10.1038/nbt.2891.

15. Jesse Cj van Dam, Jasper J Koehorst, Peter J Schaap, Vitor Ap Martins Dos Santos, and Maria Suarez-Diez. RDF2Graph a tool to recover, understand and validate the ontology of an RDF resource. Journal of biomedical semantics, 6:39, 2015. ISSN 2041-1480. doi: 10.1186/s13326-015-0038-9.

16. Christian Bizer, Tom Heath, and Tim Berners-Lee. Linked data-the story so far. Semantic services, interoperability and web applications: emerging concepts, pages 205-227, 2009.

17. Alistair Miles, Brian Matthews, Michael Wilson, and Dan Brickley. SKOS Core: Simple knowledge organisation for the Web. International Conference on Dublin Core and Metadata Applications, 0(0):3-10, September 2005. ISSN 1939-1366.

18. Grigoris Antoniou and Frank Van Harmelen. Web ontology language: Owl. In Handbook on ontologies, pages 67-92. Springer, 2004.

19. Manu Sporny, Gregg Kellogg, and Markus Lanthaler. JSON-LD 1.0 -A JSON-based Serialization for Linked Data. W3C Candidate Recommendation, 2013.

20. Oren Ben-Kiki, Clark Evans, and Ingy dot Net. YAML Ain't Markup Language (YAML) Version 1.2. yaml. org, Tech. Rep, 2009

21. Eric Prud'hommeaux, Jose Emilio Labra Gayo, and Harold Solbrig. Shape expressions: an rdf validation and transformation language. In Proceedings of the 10th International Conference on Semantic Systems, pages 32-40. ACM, 2014.

22. Timothy Lebo, Satya Sahoo, Deborah McGuinness, Khalid Belhajjame, James Cheney, David Corsar, Daniel Garijo, Stian Soiland-Reyes, Stephan Zednik, and Jun Zhao. Provo: The prov ontology. W3C recommendation, 30, 2013.

23. Frederick Giasson and Bruce D'Arcus. Bibliographic ontology. Technical report, Technical report, 2008.

24. Elvira Mitraka, Andra Waagmeester, Sebastian Burgstaller-Muehlbacher, Lynn M Schriml, Andrew I Su, and Benjamin M Good. Wikidata: A platform for data integration and dissemination for the life sciences and beyond. bioRxiv, 2015. doi: 10.1101/031971.

25. Dan Brickley and Libby Miller. Foaf vocabulary specification $0.91,2007$.

26. M Ashburner, C A Ball, J A Blake, D Botstein, H Butler, J M Cherry, A P Davis, K Dolinski, S S Dwight, J T Eppig, M A Harris, D P Hill, L Issel-Tarver, A Kasarskis, S Lewis, J C Matese, J E Richardson, M Ringwald, G M Rubin, G Sherlock, and Gene Ontology Consortium. Gene Ontology: Tool for The Unification of Biology. Nature Genetics, 25(1):25-29, 2000. ISSN 1061-4036. doi: $10.1038 / 75556$.

27. Marcus C Chibucos, Christopher J Mungall, Rama Balakrishnan, Karen R Christie, Rachael P Huntley, Owen White, Judith A Blake, Suzanna E Lewis, and Michelle Giglio. Standardized description of scientific evidence using the Evidence Ontology (ECO). Database, 2014, 2014.

28. Kirill Degtyarenko, Paula De matos, Marcus Ennis, Janna Hastings, Martin Zbinden, Alan Mcnaught, Rafael Alcántara, Michael Darsow, Mickaël Guedj, and Michael Ashburner. ChEBI: A database and ontology for chemical entities of biological interest. Nucleic Acids Research, 36(SUPPL. 1), 2008. ISSN 03051048. doi: 10.1093/nar/gkm791.

29. Rafael Alcántara, Kristian B. Axelsen, Anne Morgat, Eugeni Belda, Elisabeth Coudert, Alan Bridge, Hong Cao, Paula De Matos, Marcus Ennis, Steve Turner, Gareth Owen, Lydie Bougueleret, loannis Xenarios, and Christoph Steinbeck. Rhea - A manually curated resource of biochemical reactions. Nucleic Acids Research, 40(D1), 2012. ISSN 03051048. doi: $10.1093 / \mathrm{nar} / \mathrm{gkr} 1126$.

30. Laurel L Haak, Martin Fenner, Laura Paglione, Ed Pentz, and Howard Ratner. Orcid: a system to uniquely identify researchers. Learned Publishing, 25(4):259-264, 2012.

31. C Camacho, G Coulouris, V Avagyan, N Ma, J Papadopoulos, K Bealer, and T L Madden. BLAST+: architecture and applications. BMC Bioinformatics, 10:421, 2009. ISSN 14712105. doi: 10.1186/1471-2105-10-421.

32. Lawrence Rabiner and $B$ Juang. An introduction to hidden markov models. ieee assp magazine, 3(1):4-16, 1986.

33. Thomas Nordahl Petersen, Søren Brunak, Gunnar von Heijne, and Henrik Nielsen. Signalp 4.0: discriminating signal peptides from transmembrane regions. Nature methods, $8(10)$ : 785-786, 2011.

34. Mark A Musen. The protégé project: a look back and a look forward. Al matters, 1(4):4-12, 2015.

35. Paul Shannon, Andrew Markiel, Owen Ozier, Nitin S. Baliga, Jonathan T. Wang, Daniel 
Ramage, Nada Amin, Beno Schwikowski, and Trey Ideker. Cytoscape: A software Environment for integrated models of biomolecular interaction networks. Genome Research, 13 (11):2498-2504, 2003. ISSN 10889051. doi: 10.1101/gr.1239303.

36. Jasper J. Koehorst, Edoardo Saccenti, Peter J. Schaap, Vitor A. P. Martins dos Santos, and Maria Suarez-Diez. Protein domain architectures provide a fast, efficient and scalable alternative to sequence-based methods for comparative functional genomics. F1000Research, 5(May):1987, 2016. ISSN 2046-1402. doi: 10.12688/f1000research.9416.2.

37. Derrick E Fouts, Lauren Brinkac, Erin Beck, Jason Inman, and Granger Sutton. Panoct: automated clustering of orthologs using conserved gene neighborhood for pan-genomic analysis of bacterial strains and closely related species. Nucleic acids research, 40(22): e172-e172, 2012.

38. Li Li, Christian J Stoeckert, and David S Roos. Orthomcl: identification of ortholog groups for eukaryotic genomes. Genome research, 13(9):2178-2189, 2003.

39. Jasper J Koehorst, Jesse C J Van Dam, Ruben G A Van Heck, Edoardo Saccenti, Vitor A P Martins Dos Santos, Maria Suarez-Diez, and Peter J Schaap. Comparison of 432 Pseudomonas strains through integration of genomic, functional, metabolic and expression data. Scientific reports, 6, 2016.

40. Tjerko Kamminga, Jasper J. Koehorst, Paul Vermeij, Simen-Jan Slagman, Vitor A. P. Martins dos Santos, Jetta J. E. Bijlsma, and Peter J. Schaap. Persistence of Functional Protein Domains in Mycoplasma Species and their Role in Host Specificity and Synthetic Minima Life. Frontiers in Cellular and Infection Microbiology, 7(February):31, 2017. ISSN 22352988. doi: 10.3389/fcimb.2017.00031.

41. DigiSal, towards the digital salmon: From a reactive to a pre-emptive research strategy in aquaculture. https://www.forskningsradet.no/prosjektbanken/\#/ project/NFR/248792/Sprak=en, 2017.

42. INFECT, systems medicine to understand severe soft tissue infections. http://www . fp7infect.eu, 2017.

43. MycoSynVac, engineering mycoplasma pneumoniae as a broad-spectrum animal vaccine. http://www. mycosynvac. eu, 2017.

44. EmPowerPutida, exploiting native endowments by re-factoring, re-programming and implementing novel control loops in pseudomonas putida for bespoke biocatalysis. http: //www. empowerputida.eu, 2017.

45. Dawn Field, George Garrity, Tanya Gray, Norman Morrison, Jeremy Selengut, Peter Sterk, Tatiana Tatusova, Nicholas Thomson, Michael J Allen, Samuel V Angiuoli, Michael Ashburner, Nelson Axelrod, Frank Oliver Glöckner, Philip Goldstein, Robert Guralnick, Dan Haft, David Hancock, Henning Hermjakob, Christiane Hertz-Fowler, Phil Hugenholtz, Ian Joint, Leonid Kagan, Matthew Kane, Jessie Kennedy, Sandra Baldauf, Stuart Ballard, Guy Cochrane, James Cole, Peter Dawyndt, Paul De Vos, Paul Gilna, Frank Oliver Glöckner, Philip Goldstein, Robert Guralnick, Dan Haft, Leonid Kagan, Matthew Kane, Jessie Kennedy, George Kowalchuk, Karen Nelson, Julian Parkhill, Lita Proctor, Owen White, Andrew Spiers, Robert Stevens, Paul Swift, Chris Taylor, Adrian Tett, Sarah Turner, David Ussery, Bob Vaughan, and Naomi Ward. The minimum information about a genome sequences (MIGS) specification. Nat Biotechnol., 26(5):541-547, 2008. ISSN 1546-1696. doi: $10.1038 / 1360$.

46. Mark A Musen. The prot\{é\}g\{é\} project: a look back and a look forward. Al matters, 1(4): 4-12, 2015.

47. Philippe Rocca-Serra, Marco Brandizi, Eamonn Maguire, Nataliya Sklyar, Chris Taylor, Kimberly Begley, Dawn Field, Stephen Harris, Winston Hide, Oliver Hofmann, Steffen Neumann, Peter Sterk, Weida Tong, Susanna Assunta Sansone, and Jonathan Wren. ISA software suite: Supporting standards-compliant experimental annotation and enabling curation at the community level. In Bioinformatics, volume 27, pages 2354-2356, 2011. ISBN 1367-4803. doi: 10.1093/bioinformatics/btq415.

48. Apache Jena. Apache jena, 2013

49. Luisa Montecchi-Palazzi, Ron Beavis, Pierre-Alain Binz, Robert J Chalkley, John Cottrell, David Creasy, Jim Shofstahl, Sean L Seymour, and John S Garavelli. The psi-mod community standard for representation of protein modification data. Nature biotechnology, 26(8): 864-866, 2008.

50. Ida Schomburg, Antje Chang, Christian Ebeling, Marion Gremse, Christian Heldt, Gregor Huhn, and Dietmar Schomburg. Brenda, the enzyme database: updates and major new developments. Nucleic acids research, 32(suppl_1):D431-D433, 2004. 